\title{
A Derivation of Ad Hoc Routing Protocol from AODV using Communication Reliability
}

\author{
Dr. Pravin R. Gundalwar
}

Associate Professor, Dr. D. Y. Patil Institute of MCA, Akurdi Pune, India

\begin{abstract}
The routing protocols are playing very important job in the performance of both IP (Internet Protocol Infrastructure based) and the mobile Ad-hoc networks (MANET). The Ad hoc On Demand Distance Vector (AODV) Routing Protocol is a reactive-table driven routing protocol for mobile ad hoc networks. The route discovery and maintenance process is based on the balanced energy level in the neighbouring pair of nodes. This research is a modification on the original AODV, a basic routing protocol for its enhancement. This is an attempt to use communication reliability factor prior to establish communication using energy levels. This is examined by simulation study experimented using NS-2 on Ubantu. The performance metrics used for comparison between AODV and new protocol are PDR, routing overhead, energy level and delay.
\end{abstract}

Keywords: MANET, AODV, Routing Protocols, RREQ and RREP messages.

\section{INTRODUCTION}

Two types of wireless networks are: infrastructure oriented and infrastructure less or autonomous. The first type consists of fixed and wired gateways wherein a station communicates using access point (AP) within the coverage area for arbitrary network topology. This is shown in Fig 1. The restriction of this is that a station cannot communicate directly with any other station as the packets have to go through AP/APs before reaching to their destination. The second type has no fixed router or APs. Here the stations are not fixed rather they moves around dynamically to connect other in a coverage area. So, these stations can be called mobile stations can follow same as router in IP network. They have enough intelligence for discovery and maintenance of route establishment in the network. This is shown Fig 2. [1],[2],[5]

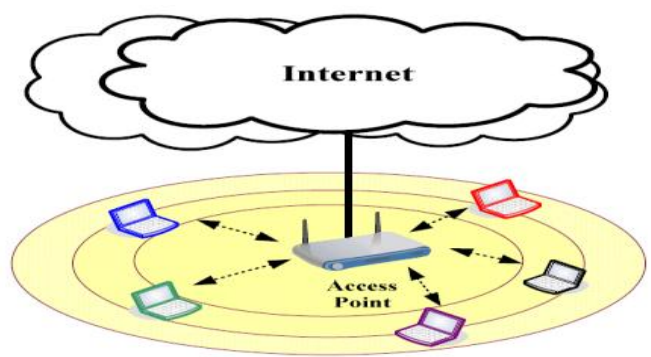

Fig. 1. Fixed Network

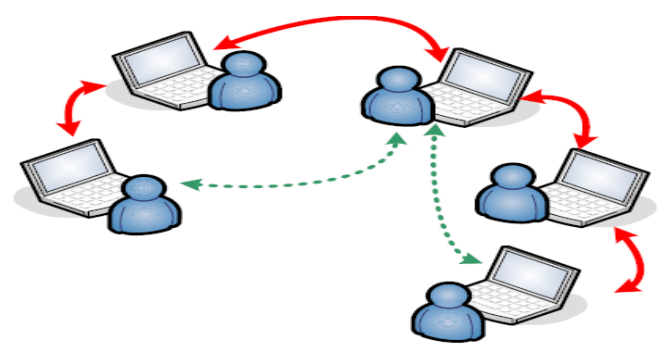

Fig. 2. Mobile Ad hoc Network
The remainder of this paper is organized as follows. In section 2, a short presentation on AODV is given. In Section III a discussion for carrying the communication reliability is introduced, whereas in Section IV simulations setup and results are presented. Finally, in Section V conclusions are drawn.

\section{AODV}

The Ad hoc on demand Distance Vector (AODV) routing protocol is one of basic routing protocols for mobile ad hoc networking. AODV is implemented with the important features or functionality used in route discovery and maintenance. It uses RREQ and RREP messages for route request and route response in route discovery mechanism while RERR route error messages, HELLO messages, and precursor lists for route maintenance very effectively.

AODV works on a very simple way for routing process. To send a message an AODV router receives a request then it checks its routing table to see if that route exists or not. The routing table entry consists of fields: destination address, next hop address, destination sequence number and hop count. In case if a route exists, the router simply forwards the message to the next hop, else it saves the message in a message queue and then it initiates a route request to determine a route.

Every node sends its neighbours periodically its whole routing table for establishing communication check if there is a useful route to another node using this neighbour as next hope. It is important to count both sent and received messages, as they will generally differ. There are two cases: first, for not all sent messages are received and second, some messages are received many times. $[3],[4],[5]$ 
Vol. 5, Issue 12, December 2016

\section{III.COMMUNICATION RELIABILITY}

The balance battery energy (B) can be found out periodically. the balance battery energy is calculated on each transmission or reception of a data packet. The B of each mobile node is calculated by,

$\mathrm{BBE}=$ Current energy - Consumed energy

Where consumed energy can be given as either equation 1 or equation 2

$$
\begin{array}{ll}
\text { Consumed energy }=\mathrm{Pt}+\mathrm{T} & \rightarrow(1) \\
\text { Consumed energy }=\mathrm{Pr}+\mathrm{T} & \rightarrow(2)
\end{array}
$$

where $\mathrm{Pt}$ is the transmitting power and $\mathrm{Pr}$ is the reception power. $\mathrm{T}$ is the transmission or reception time. The value of $\mathrm{T}$ depends on data size and data rate. A mobile node establishes communication with its neighbor mobile node. Reliability pair connects two neighbors used for establishing unicast routes [13],[15]. Reliability pair is used for determining connectivity status between a pair of mobile nodes. Reliability pair factor (RPF) [3],[16] between two nodes calculation is discussed below.

Consider the distance between any two mobile node is $\mathrm{d}$. After some time when first or second node moves in a coverage area of any one, the transmission of packets starts with reliability factor which is directly proportional to the balance battery energy.

Now, Let Ds is differential signal strength that can be calculated at either of mobile node which is inversely proportional to the distance d. Once the distance between pair neighbour nodes exceed a certain limit, the transmission signal will not be received correctly by receiver. Thus, it will result in link failureThe reliability pair factor is calculated by $\mathrm{RPF}=\mathrm{K}[\mathrm{Min}(\mathrm{B} 1, \mathrm{~B} 2)+\mathrm{Ds}] / \mathrm{d}$ where $\mathrm{K}$ is proportionality constant, $\mathrm{B} 1$ is balance battery energy at first mobile node and B2 is balance battery energy at second node. Balance battery energy of mobile node between time interval $\mathrm{t} 1$ and $\mathrm{t} 2$ is given by $\mathrm{B}(\mathrm{t} 1)=\mathrm{B}(\mathrm{t} 2)-\mathrm{P} \mathrm{t}^{*} \mathrm{~N}(\mathrm{t} 2, \mathrm{t} 1)-\mathrm{Pi}(\mathrm{t} 2, \mathrm{t} 1)$ where $\mathrm{N}$ is number of bits transmitted between internal of time. $\mathrm{Pt}$ is power required in data transmission. $\mathrm{Pi}$ is power required in internal operation performed at mobile node. If $\mathrm{F}$ be full battery energy of mobile node at time $t$ then battery energy range is given by $B R=B(t) / F(t)$. [6], [7], [8]

\section{SIMULATION AND RESULT}

This section presents the configuration of the simulation and the performance metrics of the experiments. The simulation model is developed on Ubuntu 12.04 using NS2. The simulation setup for AODV and an inventive protocol is given as follows: The topological area was $500^{2} \mathrm{~m}$. Each simulation had the duration of 500 simulated seconds with varying packet size from 64 bytes to 960 bytes. The number of mobile nodes used was 30 with speed $50 \mathrm{~m} / \mathrm{seconds}$ and pause time for 2 seconds. The number of traffic connections was 5 and its type was TCP/FTP. The other setup was standard viz Two Ray Ground radio propagation model, MAC IEEE 802.11 MAC layer protocol, Omni Antenna type, etc.
The following performance metrics were applied for comparison of the proposed approach.

Packet Delivery Ratio: It is defined as the total number of data packets received divided by total number of data packets sent at all the mobile nodes present in the simulation.

Routing Overhead: It is defined as the ratio of the AODV packets to the data packets sent and received by all the mobile nodes.

End-to-End Delay: It is defined as the delay for sending packets from source node to the fixed host.

Energy Consumption: The average of the total consumed energy of all the nodes of the network.

The comparative graph is shown in Fig 3 to 6 .

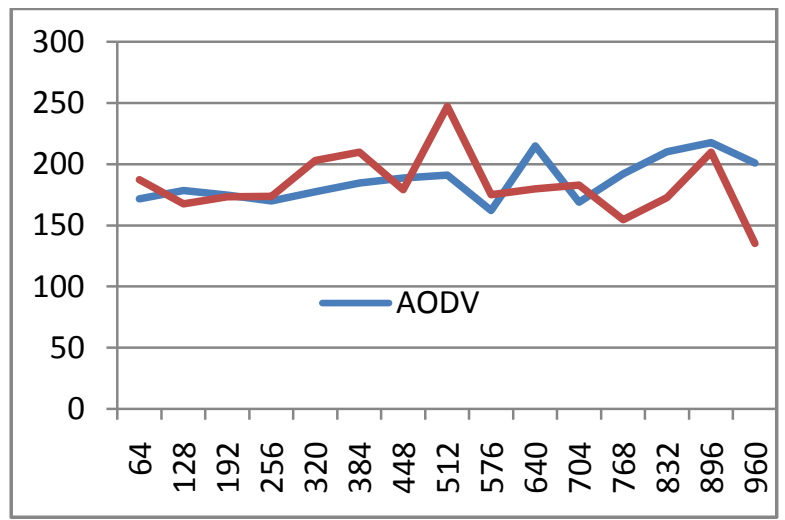

Fig. 3 A line graph for Packet Delivery Ratio Vs Packet Size (Bytes)

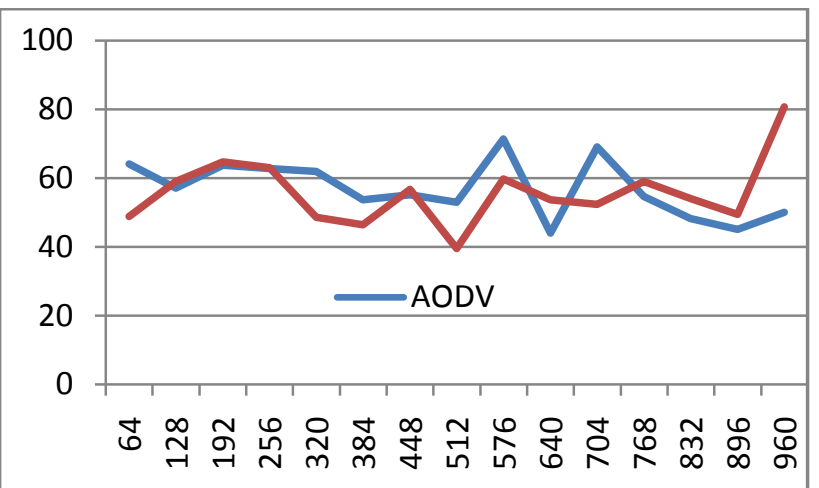

Fig. 4 A line graph for Routing Overhead Vs Packet Size (Bytes)

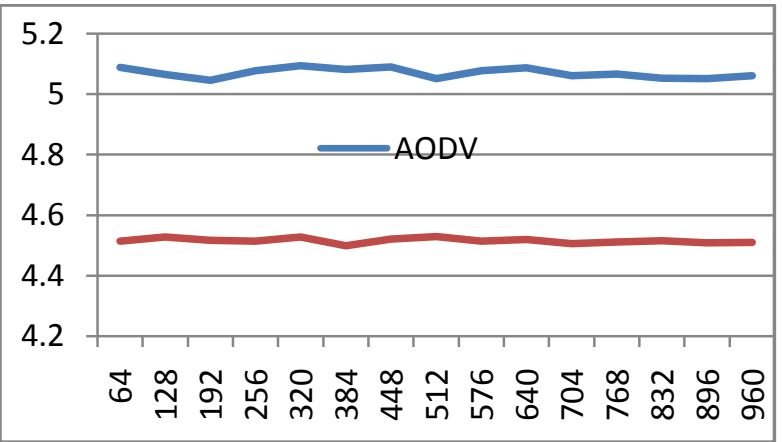

Fig. 5 A line graph for End-to-End Delay Vs Packet Size (Bytes) 
Vol. 5, Issue 12, December 2016

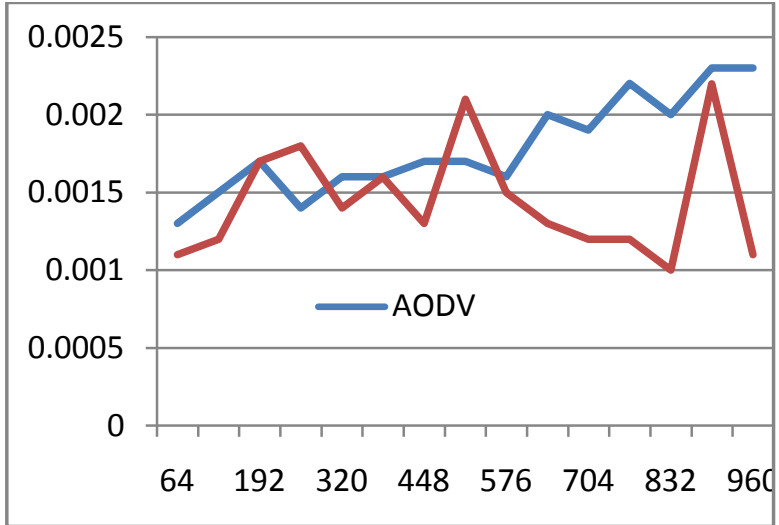

Fig. 6 A line graph for Energy Consumption Vs Packet Size (Bytes)

\section{CONCLUSION}

Energy is out most important for communication reliability in ad hoc mobile network. One strongly interpreted that the mobile ad hoc network is completely dependent on node energy level. It is observed that all metrics used for experimentation viz, the packet delivery rate, routing overhead, energy consumption, and end-toend delay performance of new inventing routing protocol is performed superior to AODV routing protocol. Thus, new routing protocol can be implemented for achieving communication reliability. AODV does not retransmit data packets that are lost due to low battery energy level and hence does not guarantee packet delivery which can be overcome in the new invented protocol. Finally, one can design and derive innovative properties for more efficient protocol for MANET.

\section{ACKNOWLEDGMENT}

The author is thankful to Dr. Vinay N. Chavan, Associate Professor \& Head, Department of Computer Science, S. K. Porwal College, Kamptee, Nagpur (MS) India for his valuable guidance and support.

\section{REFERENCES}

[1] Clifton Lin, "AODV Routing Implementation for Scalable Wireless Ad Hoc Network Simulation (SWANS): http://jist.ece.cornell.edu/ docs/040421-swans-aodv.pdf

[2] Rainer Bauman, A ODV, Presentation at ETH Zürich April 02: http://www.rainer-baumann.ch/public/qec.pdf

[3] Charles E Perkins, Elizabeth M Royer, "Ad hoc On Demand Distance Vector Routing": https://www.ee.iitb.ac.in/ comlab/ seminar/nishant1_Ref1.pdf

[4] Preeti Sachan and Pabitra Mohan Khilar, Securing AODV Routing Protocol in MANET Based on Cryptographic Authentication Mechanism, International Journal of Network Security \& Its Applications (IJNSA), Vol.3, No.5, Sep 2011

[5] Wadhah Al-Mandhari, Koichi Gyoda, Nobuo Nakajima, "Ad-hoc On Demand Distance Vector (AODV) Performance Enhancement with Active Route Time-Out parameter", WSEAS TRANSACTIONS on COMMUNICATIONS Manuscript received Feb 12, 2008; revised Aug 10, 2008

[6] Baisakh, Nileshkumar R. Patel "Energy Saving and Survival Routing Protocol for Mobile Ad Hoc Networks", International
Journal of Computer Applications (0975-888) Volume 48-No.2, June 2012

[7] Surabhi Purwar1, Shiva Prakash, "Reliable pair protocol for Link Stability in MANET", IRACST - International Journal of Computer Networks and Wireless Communications (IJCNWC), ISSN: 2250-3501, Vol.2, No.3, June 2012

[8] Rajashekhar C. Biradar, Sunilkumar S. Manvi, "Information Priority Based Multicast Routing in MANETs", International Journal of Wireless \& Mobile Networks (IJWMN) Vol. 3, No. 3, June 2011

\section{BIOGRAPHY}

Dr. Pravin R. Gundalwar, is Associate Professor at Dr. D. Y. Patil Institute of MCA, Akurdi, Pune (MS), India. $\mathrm{He}$ received $\mathrm{PhD}$ (Computer Science) from R.T.M. Nagpur University, Nagpur and MCA (Faculty of Engineering \& Technology) from Amravati University, Amravati. His interests are in Computer Network, Network Security and Optimization Techniques. 